\title{
Prevalencia de malformaciones congénitas en hijos de madres mayores de 34 años y adolescentes. Hospital Clínico de la Universidad de Chile, 2002-201 1
}

\author{
Julio Nazer Herrera. ${ }^{1}$, Lucía Cifuentes Ovalle. ${ }^{2}$ \\ ${ }^{1}$ Maternidad, Hospital Clínico de la Universidad de Chile. ${ }^{2}$ Programa de Genética Humana, Instituto de Ciencias \\ Biomédicas, Facultad de Medicina. Universidad de Chile.
}

\section{RESUMEN}

Antecedentes: Las madres mayores de 34 años han aumentado en Chile. La mayor edad materna aumenta el riesgo de malformaciones congénitas (MFC) Objetivos: Determinar la tasa de prevalencia de MFC entre adolescentes y mayores de 34 años. Método: Se utilizó la base de datos de la Maternidad del Hospital Clínico Universidad de Chile (2002-2011). Se estudió todos los nacimientos, vivos, mortinatos y malformados de 500 gramos o más, de madres menores de 20 y mayores de 34 años. Se calculó y comparó las tasas de prevalencia al nacer de todas las malformaciones de cada grupo etario. Resultados: Hubo 15.636 nacimientos, 0,98\% fueron mortinatos, $7,5 \%$ hijos de madres menores de 20 años y $27,7 \%$ de mayores de 34 años. La tasa global de MFC fue 8,8\%; 7\% en menores de 20 años y 9,6\% en mayores de 34 años ( $p=0,007)$. Síndrome de Down fue 12 veces más frecuente en los hijos de madres mayores de 34 años que en las adolescentes. Las mayores de 34 años presentaron significativamente más mortineonatalidad en sus hijos malformados que las adolescentes, pero menor frecuencia de bajo peso, menor consumo de tabaco, alcohol y mariguana. Conclusión: El grupo de madres mayores de 34 años es significativamente diferente al grupo de adolescentes, tanto en prevalencia de MFC como en mortalidad neonatal de malformados, bajo peso al nacer y hábitos de vida.

\section{PALABRAS CLAVES: Malformaciones congénitas, mortalidad perinatal, embarazo adolescente, edad materna avanzada}

\section{SUMMARY}

Background: Mothers older than 34 years have increased trend in Chile. The maternal age increases the risk of congenital malformations (CMF). Objectives: Determine the prevalence rate of CMF among newborn of teenagers mothers and older than 34 years old. Method: We used the database of the Maternity Hospital, University of Chile (2002-2011). We studied all births, live, stillbirths and malformed newborn of 500 grams or more, from mothers under 20 and over 34 years. We calculated and compared the birth prevalence rates of all malformations for each age group. Results: There were 15,636 births, $0.98 \%$ were stillbirths, $7.5 \%$ children of mothers under 20 years and $27.7 \%$ older than 34 years. The overall rate of CMF was $8.8 \% ; 7 \%$ in under 20 years and $9.6 \%$ in mothers over 34 years $(p=0.007)$. Down syndrome was 12 times more common in children of mothers older than 34 years than in adolescents. The older than 34 years had significantly more neonatal mortality in malformed children than adolescents, but lower frequency of low birth weight, lower consumption of snuff, alcohol and marijuana. Conclusion: The group of mothers older than 34 years is significantly different from the group of teenagers, both in prevalence of CMF as neonatal mortality, low birth weight and lifestyle habits.

KEY WORDS: Congenital malformation, perinatal mortality, adolescent pregnancy, advanced maternal age 


\section{INTRODUCCIÓN}

Las malformaciones congénitas son una de las principales causas de mortalidad perinatal e infantil. En Chile su prevalencia al nacimiento es de 3,1\% (1), tasa superior a todos los países sudamericanos, con excepción de Brasil.

En la última publicación nuestra (2), mostramos como en Chile el grupo de madres mayores de 34 años ha aumentado más de $50 \%$ al comparar los datos proporcionados por el Ministerio de Salud de Chile (3) del período 1990-2008, y más de 120\% en el Hospital Clínico de la Universidad de Chile (HCUCH) entre 1982 y 2010. Esto se asoció a una significativa mayor frecuencia de prematuros, de malformaciones congénitas (MFC), de síndrome de Down y otras cromosomopatías; de recién nacidos femeninos y de más partos por cesárea; por el contrario, disminuyeron los casos de restricción del crecimiento intrauterino y de recién nacidos pequeños para la edad gestacional.

Estos hallazgos corroboran lo encontrado anteriormente por nosotros $(2,4-7)$, por otros autores nacionales $(8,9,10)$ y autores extranjeros $(11-16)$. También se ha comunicado un riesgo aumentado para otras malformaciones, especialmente defectos de cierre del tubo neural, anencefalia y espina bífida en hijos de madres mayores de 34 años (17).

El objetivo principal de este estudio es establecer la prevalencia al nacimiento de las MFC, detectar cuales se presentan con mayor frecuencia entre los hijos de madres mayores de 34 años, compararlas con el grupo de hijos de adolescentes, y estudiar los factores de riesgo que pudieran afectar a ambos grupos.

\section{PACIENTES Y MÉTODO}

Se analizó la base de datos del ECLAMC del Hospital Clínico de la Universidad de Chile (HCUCH), analizándose el período de 10 años comprendido entre enero de 2002 y diciembre de 2011. Se estudió todos los nacimientos, vivos, mortinatos y malformados de 500 gramos o más, de madres menores de 20 y mayores de 34 años.

En cada grupo se individualizaron todas las MFC, se calculó el porcentaje que cada una representa dentro del total y se agruparon por sistemas. Se estudiaron otras variables del recién nacido (RN), como peso de nacimiento, edad gestacional, prematuridad y bajo peso de nacimiento, $\mathrm{y}$ variables maternas como edad, hábitos de vida (consumo de alcohol, tabaquismo y drogas). Se comparó las ta- sas de prevalencia al nacer de las malformaciones congénitas y los antecedentes del grupo de madres estudiadas con prueba del Chi cuadrado o prueba de Fisher en los casos de bajo número de casos. Se establece como significativo un valor $p<0,05$.

\section{RESULTADOS}

En el período estudiado ocurrieron 15.636 nacimientos consecutivos: 15.483 nacidos vivos (NV) y 153 mortinatos (NM). Esto representa una tasa de mortinatalidad de 9,88/1000 NV.

Hubo 3.399 RN $(27,7 \%)$ hijos de madres mayores de 34 años, 3.358 fueron NV $(98,8 \%)$ y 41 NM $(1,2 \%)$. De las madres adolescentes hubo 1.174 RN, 1.162 NV $(98,98 \%)$ y 12 NM $(1,02 \%)$.

En el total de nacimientos 1.376 RN presentaban una o más malformaciones congénitas $(8,8 \%)$ (Tabla I). En el grupo de madres mayores de 34 años hubo $326 \mathrm{RN}$ malformados $(9,6 \%)$, cifra significativamente mayor que la frecuencia encontrada en madres adolescentes $(7,0 \% ; p=0,023)$. De ellos 311 eran NV y 15 NM (4,6\%; 15/326). De los nacidos vivos malformados fallecieron 15 , que representa una mortalidad neonatal en malformados de $4,8 \%(15 / 311)$, mayor que la mortalidad neonatal de malformados en hijos de madres adolescentes $(2,4 \% ; p=0,004)$. En el grupo de hijos de madres adolescentes se encontró 82 malformados, con una tasa de $7 \%(p=0,007)$ (Tabla I).

La cardiopatía congénita fue el diagnóstico más frecuente $(15,3 \%)$, seguido por el síndrome de Down (12\%). Al agrupar las malformaciones por sistemas (Tabla II) apreciamos que las anomalías de piel y fanerios son las más frecuentes $(23,4 \%)$, seguida de las cardiovasculares $(16,9 \%)$, cromosómicas $(16,1 \%)$ y genitourinarias $(15 \%)$.

En la Tabla III presentamos una comparación de varios factores demográficos entre los $\mathrm{RN}$ malformados de los dos grupos en estudio. Apreciamos que son diferentes en su composición: el grupo de madres mayores de 34 años tienen significativamente más $R N$ malformados $(p=0,0069)$, más RN grandes para la edad gestacional (GEG) $(p<0,0001)$. Por el contrario tienen menor frecuencia de niños de bajo peso al nacer $(p<0,0001)$, y menor consumo de alcohol, tabaco y mariguana $(p<0,0001)$.

En las madres mayores de 34 años, en 128 casos existía el antecedente de otro malformado en la familia $(3,8 \%)$, mientras que 30 adolescentes $(2,6 \%)$ tienen ese antecedente. No hubo casos de consanguinidad. 
Tabla I

PREVALENCIA AL NACIMIENTO DE MALFORMACIONES CONGÉNITAS EN HIJOS DE MADRES ADOLESCENTES Y MAYORES DE 34 AÑOS

\begin{tabular}{lccr}
\hline Grupo etario & $\begin{array}{c}\text { Nacimientos } \\
(\mathrm{n})\end{array}$ & $\begin{array}{c}\text { Malformados } \\
(\mathrm{n})\end{array}$ & Frecuencia * (\%) \\
\hline$<20$ años & 1.174 & 82 & 7,0 \\
$>34$ años & 3.399 & 326 & 9,6 \\
Todos & 15.636 & $1.376^{\star \star}$ & 8,8
\end{tabular}

* $\mathrm{p}=0,0069 ;{ }^{* *}$ una o más malformación.

Tabla II

MALFORMACIONES CONGÉNITAS POR SISTEMAS EN HIJOS DE MADRES MAYORES DE 34 AÑOS

\begin{tabular}{lcc}
\hline Malformaciones $^{*}$ & $\mathrm{n}$ & $\begin{array}{c}\text { Frecuencia } \\
\text { (\%) }\end{array}$ \\
\hline Piel y fanereos & 83 & 23,4 \\
Cardiovaculares & 60 & 16,9 \\
Cromosómicas & 57 & 16,1 \\
Genitourinarias & 53 & 15,0 \\
Esqueléticas & 38 & 10,7 \\
Digestivas & 17 & 4,8 \\
Sistema nervioso central & 16 & 4,5 \\
Sindrómicas & 8 & 2,3 \\
Tumorales & 3 & 0,8 \\
Otras & 19 & 5,4 \\
Total & 354 & 99,9 \\
\hline
\end{tabular}

*326 malformados (una o más malformación).

\section{Tabla III \\ COMPARACIÓN DE ALGUNOS FACTORES ENTRE RECIÉN NACIDOS DE MADRES MENORES DE 20 Y MAYORES DE 34 AÑOS}

\begin{tabular}{|c|c|c|c|}
\hline Variable & $\begin{array}{c}<20 \text { años } \\
(\%)\end{array}$ & $\begin{array}{l}>34 \text { años } \\
(\%)\end{array}$ & $\begin{array}{l}\text { Valor } \\
\mathrm{p}\end{array}$ \\
\hline Mortinatos & 1,0 & 1,2 & $0,6011^{*}$ \\
\hline Mortineonatos & 0,1 & 0,4 & $0,18^{*}$ \\
\hline Malformados & 7,0 & 9,6 & 0,0069 \\
\hline $\begin{array}{l}\text { Adecuados para la edad } \\
\text { gestacional }\end{array}$ & 78,0 & 72,1 & 0,0001 \\
\hline $\begin{array}{l}\text { Pequeños para la edad } \\
\text { gestacional }\end{array}$ & 15,9 & 16,9 & $0,41^{*}$ \\
\hline $\begin{array}{l}\text { Grande para la edad } \\
\text { gestacional }\end{array}$ & 6,1 & 10,7 & 0,0001 \\
\hline Bajo peso al nacer & 24,4 & 16,3 & 0,0001 \\
\hline Prematuros & 23,2 & 25,5 & $0,11^{*}$ \\
\hline Consumo de alcohol & 34,1 & 26,4 & 0,0001 \\
\hline Consumo de mariguana & 18,3 & 0 & 0,0001 \\
\hline Consumo de tabaco & 39,0 & 25,5 & 0,0001 \\
\hline
\end{tabular}

*no significativo $(p>0,05)$

adolescentes $(p<0,00075)$, al igual que cardiopatías congénitas $(p=0,038)$. Trisomía $13(p=0,069)$ y $18(p=0,23)$ fueron más frecuentes en este grupo, pero sin diferencias significativas. Por el contrario, fosita pilonidal y hemangiomas se presentan, con una prevalencia mayor en los hijos de madres adolescentes, la misma tendencia muestran gastrosquisis, holoprocencefalia, luxación de cadera y ano imperforado sin presentar diferencias significativas ( $p=0,065, p=0,065$ y $p=0,054$ respectivamente). 
Tabla IV a

PREVALENCIA AL NACER DE MALFORMACIONES CONGÉNITAS EN HIJOS DE MADRES MAYORES DE 34 Y MENORES DE 20 AÑOS

\begin{tabular}{|c|c|c|c|c|c|}
\hline Diagnóstico & $<20$ años & Tasa & >34 años & Tasa & Valor $p$ \\
\hline Nacimientos & 1.174 & - & 3.399 & - & - \\
\hline Malformados & 82 & 7,0 & 326 & 9,6 & 0,007 \\
\hline Agenesia cuerpo calloso & 1 & 0,9 & - & - & $0,25^{\star}$ \\
\hline Agenesia renal & - & - & 1 & 0,3 & $0,74^{*}$ \\
\hline Anencefalia & - & - & 1 & 0,3 & $0,74^{*}$ \\
\hline Ano imperforado & 3 & 2,6 & 1 & 0,3 & $0,054^{*}$ \\
\hline Aplasia cutis & 2 & 1,7 & 2 & 0,6 & $0,28^{*}$ \\
\hline Arteria umbilical única & 2 & 1,7 & 7 & 2,1 & $0,58^{\star}$ \\
\hline Atresia esófago & 1 & 0,9 & 3 & 0,9 & $0,72^{*}$ \\
\hline Atresia yeyuno-ileal & - & - & 1 & 0,3 & $0,74^{*}$ \\
\hline Cardiopatía congénita & 8 & 6,8 & 50 & 14,7 & 0,037 \\
\hline Criptorquidia & 3 & 2,6 & 17 & 5,0 & $0,27^{*}$ \\
\hline Displasia esquelética & 1 & 0,9 & - & - & $0,25^{\star}$ \\
\hline Displasia renal & 1 & 0,9 & - & - & $0,25^{\star}$ \\
\hline Fístula auris & 3 & 2,6 & 15 & 4,4 & $0,28^{*}$ \\
\hline Fisura palatina & 1 & 0,9 & 1 & 0,3 & $0,45^{\star}$ \\
\hline Fosita pilonidal & 7 & 6,0 & 7 & 2,1 & 0,044 \\
\hline Gastrosquisis & 2 & 1,7 & - & - & $0,065^{\star}$ \\
\hline Hemangioma & 7 & 6,0 & - & - & 0,0001 \\
\hline Hidronefrosis & - & - & 4 & 1,2 & $0,31^{*}$ \\
\hline
\end{tabular}

*no significativo $(p>0,05)$. Tasa: x 1000 NV. Total de nacimientos del período: 15.636

Tabla IV b

PREVALENCIA AL NACER DE MALFORMACIONES CONGÉNITAS EN HIJOS DE MADRES MAYORES DE 34 Y MENORES DE 20 AÑOS

\begin{tabular}{|c|c|c|c|c|c|}
\hline Diagnóstico & $<20$ años & Tasa & > 34 años & Tasa & Valor $p$ \\
\hline Hidrops & 1 & 0,9 & 3 & 0,9 & $0,72^{*}$ \\
\hline Hiperplasia suprarrenal & 1 & 0,9 & 1 & 0,3 & $0,45^{\star}$ \\
\hline Holoprocencefalia & 2 & 1,7 & - & - & $0,065^{*}$ \\
\hline Ictiosis & 1 & 0,9 & - & - & $0,25^{\star}$ \\
\hline Labio leporino & - & - & 7 & 2,1 & $0,125^{*}$ \\
\hline Luxación de cadera & 4 & 3,4 & 7 & 2,1 & $0,31^{*}$ \\
\hline Malformación de dedos/pies & 3 & 2,6 & 2 & 0,6 & $0,11^{*}$ \\
\hline Micrognatia & - & - & 16 & 4,7 & 0,009 \\
\hline Microtia & 2 & 1,7 & 5 & 1,5 & $0,57^{*}$ \\
\hline Nevo pigmentado & 9 & 7,7 & 37 & 10,9 & $0,32^{*}$ \\
\hline Nevo sebáceo & 2 & 1,7 & 4 & 1,2 & $0,48^{*}$ \\
\hline Papiloma preauricular & 4 & 3,4 & 15 & 4,6 & $0,44^{*}$ \\
\hline Piebaldness & 1 & 0,9 & - & - & $0,25^{*}$ \\
\hline Pie Bott & 8 & 6,8 & 21 & 6,2 & $0,81^{*}$ \\
\hline Pielectasia renal & 3 & 2,6 & 6 & 1,8 & $0,42^{*}$ \\
\hline Polidactilia & 1 & 0,9 & 4 & 1,2 & $0,61^{*}$ \\
\hline Quiste de ovario & 1 & 0,9 & 3 & 0,9 & $0,72^{*}$ \\
\hline Síndrome de Down & 1 & 0,9 & 39 & 11,5 & 0,0009 \\
\hline Otros síndromes & 1 & 0,9 & 4 & 1,2 & $0,61^{*}$ \\
\hline Trisomía 18 & 1 & 0,9 & 9 & 2,6 & $0,32^{*}$ \\
\hline Trisomía 13 & - & - & 9 & 2,6 & 0,069 \\
\hline Ventriculomegalia & 1 & 0,9 & 2 & 0,6 & $0,50^{*}$ \\
\hline
\end{tabular}

*no significativo ( $p>0,05)$. Tasa: x 1000 NV. Total de nacimientos del período: 15.636 


\section{DISCUSIÓN}

En nuestro estudio se pudo comprobar que la prevalencia de MFC en hijos de mujeres mayores de 34 años fue significativamente mayor que en los hijos de madres adolescentes.

La prevalencia de MFC por grupos etarios es diferente a la nacional porque primero, el $\mathrm{HCUCH}$ es un Centro de referencia para patologías complejas, tanto maternas como fetales; segundo, la distribución etaria de las madres es muy diferente a la nacional. En el $\mathrm{HCUCH} 7,5 \%$ son menores de 20 años y $21,7 \%$ mayores de 34 años (2). Esta distribución es diferente a la que da el MINSAL para el total de Chile en el período 1990-2008: 15,2\% para las madres adolescentes y $13,8 \%$ para las mayores de 34 años (3). Esto demuestra que la población atendida en el $\mathrm{HCUCH}$ es diferente a la atendida en los hospitales públicos del Servicio Nacional de Salud, lo que de algún modo tendrá que influir en las tasas de prevalencia al nacimiento de MFC. Es conocido el hecho que la edad materna avanzada es un factor de riesgo para MFC $(2,4-7)$.

Algunos autores han demostrado tasas mayores en las edades extremas, adolescentes y mayores de 34 años $(6,17-20)$, y se describe una distribución en "J" o en "U"; y tercero, a que el registro, que comenzó en 1969, incorpora a todas las malformaciones, mayores y menores en $\mathrm{RN}$ vivos y mortinatos, lo que no ocurre en los hospitales públicos con gran presión asistencial.

La tasa de prevalencia al nacimiento de MFC en el $\mathrm{HCUCH}$ fue de $8,5 \%$ para el período 1998-2010 (21-22), en esta muestra encontramos una tasa de $8,8 \%$. Sin embargo, estas tasas son muy diferentes al comparar las adolescentes con las mayores de 34 años. En el grupo de mujeres mayores de 34 años hubo una significativa mayor prevalencia de MFC en sus hijos que el grupo de las madres adolescentes. Entre los hijos de madres mayores obtuvimos tasas significativamente más altas $(9,6 \%$ contra $7 \%$ en adolescentes). Esto último podría ser explicado por el número bajo de nacimientos en el grupo de madres adolescentes, que corresponde a la cuarta parte del grupo de madres mayores de 34 años.

Las tasas encontradas en el $\mathrm{HCUCH}$ han sido tradicionalmente mayores que en las encontradas en otros hospitales chilenos del ECLAMC $(21,22,23)$ lo que se podría explicar por ser un hospital de alta complejidad y referente para patologías maternas y fetales importantes. Las tasas de prevalencia al nacimiento para Chile en el período 1995-2008 (22) fue de $3,1 \%$ y para el total del ECLAMC de $2,6 \%$.

Si comparamos la prevalencia de las malformaciones en los dos grupos encontramos algunos hechos interesantes, como que algunas malformaciones se presentan con mayor frecuencia en uno o el otro grupo. Ya conocíamos el hecho de que el síndrome de Down es significativamente más frecuente entre los hijos de madres de mayor edad (3-6). Otras aneuploidías como trisomía 13 y 18 son también más frecuentes en ese grupo, aunque las diferencias no fueron significativas. Por el contrario, hay otras anomalías que se presentan con mayor frecuencia entre los hijos de las adolescentes y muy raras veces en los hijos de las mayores de 34 años, como gastrosquisis, ano imperforado, hemangiomas y fosita pilonidal, que estuvieron al borde de la significancia estadística.

Cuando comparamos otros factores, también encontramos diferencias significativas entre adolescentes y madres mayores. El porcentaje de niños de bajo peso de nacimiento es significativamente menor en las mayores de 34 años que en el grupo de adolescentes $(p<0,00001)$. En mortineonatalidad de RN malformados ocurre algo similar $(p<0,0004)$. Mientras que prematuridad es mayor en el grupo de $\mathrm{RN}$ de madres mayores, pero sin ser estadísticamente significativo (Tabla III).

No encontramos diferencias significativas en el antecedentes de otro malformado en la familia, ni consanguinidad, pero sí una tendencia no significativa a mayor frecuencia de metrorragia en el primer trimestre del embarazo (0,97\%), en las madres mayores que en las adolescentes $(0,43 \% ; p=0,076)$.

En hábitos de vida el comportamiento del grupo de madres adolescentes es significativamente diferente, tienen mayor frecuencia de consumo de alcohol y drogas que las madres mayores. Existen numerosas publicaciones que demuestran la importancia que tienen como factores de riesgo para MFC, el consumo de alcohol, tabaco y drogas (24-27).

\section{CONCLUSIÓN}

El grupo etario de madres mayores de 34 años presenta una significativa mayor tasa de prevalencia al nacimiento de MFC comparadas con las madres adolescentes. Mayor mortalidad perinatal en malformados y menor porcentaje de RN de bajo peso. Entre las madres adolescentes se observa mayor consumo de tabaco, alcohol y mariguana.

\section{REFERENCIAS}

1. Nazer J, Cifuentes L. Malformaciones congénitas en Chile y Latino América: Una visión epidemiológica del ECLAMC del período 1995-2008. Rev Med Chile 2011;139:72-8.

2. Nazer J, Ramírez C, Cifuentes L, Águila A, Gutiérrez R. Cambios en la distribución etaria de las madres en Chile y en el Hospital Clínico de la Universidad de Chile y su influencia en la morbimortalidad neonatal. Rev Chil Obstet Ginecol 2012;77:183-9.

3. MINSAL. DEIS. Nacidos vivos inscritos, según estado civil y edad de la madre, por Región. Chile 2000-2009. Disponible en: http://deis.minsal.cl/deis/salidas08/ nac/Nacidos_Vivos_edad_estado_civil_madre_ regi\%C3\%B3n_Chile2000_2009.htm. 
4. Nazer J, Cifuentes L. Estudio epidemiológico global del síndrome de Down. Rev Chil Pediatr 2011;82:105-12.

5. Nazer J, Cifuentes L, Águila A, Ureta P, Bello MP, Melibosky R. Edad materna y malformaciones congénitas. Un registro de 35 años: 1990- 2004. Rev Med Chile 2007;135:1463-9.

6. Nazer J, Águila A, Cifuentes L. Vigilancia epidemiológica del Síndrome de Down en Chile: 1972-2005. Rev Med Chile 2006;134:1459-57.

7. Nazer J, Eagling MA, Cifuentes L. Incidencia del síndrome de Down en la maternidad del Hospital Clínico de la Universidad de Chile. Un registro de 25 años. Rev Med Chile 1998;126:383-90.

8. Donoso E, Carvajal J, Domínguez MA. Reducción de la fecundidad y envejecimiento de las mujeres chilenas en edad fértil: 1990-2004. Rev Med Chile 2009;137:766-90.

9. Chamy V, Cardemil F, Betancour P, Ríos M, Leighton L. Riesgo obstétrico y perinatal en embarazadas mayores de 35 años. Rev Chil Obstet Ginecol 2009;74:331-8.

10. Donoso E, Villarroel del Pozo L. Edad avanzada y riesgo reproductivo. Rev Med Chile 2003;131:55-9.

11. Tipiani-Rodríguez $O$. Es la edad avanzada un factor de riesgo independiente para complicaciones maternoperinatales? Rev Per Ginecol Obstet 2006;52:179-85.

12. Reefhuis J, Honein M. Maternal age and non-chromosomal birth defects, Atlanta--1968-2000: teenager or thirty-something, who is at risk? Birth Defects Res A Clin Mol Teratol 2004;70:572-9.

13. Sherman SL, Freeman SB, Allen EG, Lamb NE. Risk factors for non disjunction of Trisomy. Citogenet Genom Res 2005;111:273-80.

14. Cocchi G, Gualdi S, Bower C, Halliday J, Jonsson B, Myrelid $A$, et al. International trends of Down syndrome 1993-2004: Births in relation to maternal age and terminations of pregnancies. Birth Defects Res A Clin Mol Teratol 2010;88:474-9.

15. Hollier LM, Leveno KJ, Kelly MA, McIntire DD, Cunningham FG. Maternal age and malformations in sin- gleton births. Obstet Gynecol 2000;96:701-6.

16. Baid PA, Sudovnik AD, Yee Im. Maternal age and birth defects: a population study. Lancet 1991;337:527-30.

17. Vieira A, Castillo S. Edad materna y defectos del tubo neural: evidencia para un efecto mayor de espina bífida que anencefalia. Rev Med Chile 2005;133:62-70.

18. Kazaura MR, Lie RT, Irgens LM, Didriksen A, Kapstad $M$, Egenaes J, Bjerkedal T. Increasing risk of gastroschisis in Norway: an age-period-cohort analysis. Am J Epidemiol 2004;159:358-63.

19. Lubinsky MS. Association of prenatal vascular disruptions with decrease maternal age. Am J Med genet 1997;69:237-9.

20. Frasier AM, Brockert JE, Ward RH. Association of young maternal age with adverse reproductive outcomes. N Engl 1995;332:1113-7.

21. Nazer J, Cifuentes L, Ramírez C. Malformaciones urinarias del recién nacido. Estudio ECLAMC 19982010. Rev Chil Pediatr 2011;82:512-9.

22. Nazer J, Ramírez C, Cifuentes L. Atresia de esófago y sus asociaciones preferenciales. Rev Chil Pediatr 2011;82:36-42.

23. Nazer J, Cifuentes L. Malformaciones congénitas en Chile y Latino América: Una visión epidemiológica del ECLAMC del período 1995-2008. Rev Med Chile 2011;139:72-8.

24. Morales-Suárez M, Mille $C$, Christensen K. Smoking habits, nicotina use and congenital malformations. Obstet Gynecol 2006;107:51-7.

25. Baumann P, Schild C, Hume RF, Sokol RJ. Alcohol abuse. A persistent preventable risk for congenital anomalies. Int J Gynecol Obstet 2006;95:66-72.

26. Woods S, Raju U. Maternal smoking and the birth defects: a cohort study. J Am Board Fam Pract 2001;14:330-4.

27. Behnke M, Eyler FD, Garvan CW, Wobie K. The search for congenital malformations in newborns with fetal cocaine. Pediatrics 2001;107;e74. Disponible en: http://pediatrics.aappublications.org/content/107/5/ e74.full.pdf+html. 